\title{
Carnets
}

Revue électronique d'études françaises de l'APEF

Deuxième série - 20 | 2020

Imaginaire(s) du Voyage

\section{Grèce rêvée, Grèce vécue : étude croisée des récits de voyage de Jacques Lacarrière et Zbigniew Herbert}

Madeleine Séguier

\section{(2) OpenEdition}

Journals

Édition électronique

URL : http://journals.openedition.org/carnets/12147

DOI : 10.4000/carnets. 12147

ISSN : 1646-7698

Éditeur

APEF

Référence électronique

Madeleine Séguier, «Grèce rêvée, Grèce vécue : étude croisée des récits de voyage de Jacques Lacarrière et Zbigniew Herbert », Carnets [En ligne], Deuxième série - 20 | 2020, mis en ligne le 30 novembre 2020, consulté le 19 avril 2021. URL : http://journals.openedition.org/carnets/12147 ; DOI https://doi.org/10.4000/carnets.12147

Ce document a été généré automatiquement le 19 avril 2021.

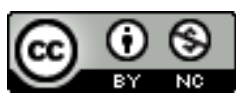

Carnets est mis à disposition selon les termes de la licence Creative Commons - Atribution - Pas d'utilisation commerciale 4.0 International. 


\title{
Grèce rêvée, Grèce vécue : étude croisée des récits de voyage de Jacques Lacarrière et Zbigniew Herbert
}

\author{
Madeleine Séguier
}

De Virgile à Paul Valéry, [la tradition grecque]

leur a ouvert à tous la porte d'un pays assez vaste

pour que chacun y ait sa province, assez désert

pour s'y promener nu, peuplé pourtant de

fantômes qui chantent.

Yourcenar, 1989 : 32 .

1 Dans ce passage de l'article "Mythologie grecque et mythologie de la Grèce ", Marguerite Yourcenar joue sur l'ambiguïté de la représentation de la Grèce : le " pays » évoqué ici n'est pas la Grèce en tant qu'État-nation précisément délimitée dans l'espace, mais bien une contrée imaginaire forgée par la réception occidentale de la culture grecque antique. La métaphore géographique vient avant tout donner corps à une longue tradition littéraire qui, de l'Antiquité romaine à nos jours, s'est approprié cette culture grecque antique, déformant ou occultant parfois les lieux qui l'ont vu naître. Le "pays " imaginaire dont chaque auteur trace les contours à sa guise se superpose pourtant lointainement à un pays réel. Rarement visité alors même qu'il est perçu par les européens comme le berceau de leur culture, cet espace aux frontières mouvantes connaît un regain d'intérêt avec l'avènement du romantisme du début du $\mathrm{XIX}^{\mathrm{e}}$ siècle qui voit naître la mode du voyage - et du récit de voyage - en Orient. La Grèce constitue alors une étape sur ce voyage, avant de devenir une destination à part entière après la guerre d'Indépendance (1821-1830) qui suscite un fort élan philhellène dans toute l'Europe, et en particulier dans les deux pays qui intéressent cette étude: la France et la Pologne. Les écrits qui en sont issus jouent ainsi avec une double tradition littéraire : celle du récit de voyage et celle des représentations de la Grèce. L'une des caractéristiques du genre consiste à mettre en relation la "province » dans laquelle 
l'écrivain se promenait "nu», avec la terre grecque qu'il arpente au cours de son voyage. Cette expérience parfois déroutante de la rencontre entre l'imaginaire et le réel est abondamment décrite par Jacques Lacarrière dans l'Eté grec (1976) et par Zbigniew Herbert dans Le labyrinthe au bord de la mer (2000, publication posthume). Témoins de la prégnance du récit de voyage en Grèce jusque dans la seconde moitié du $\mathrm{xx}^{\mathrm{e}}$ siècle, ces deux voyageurs européens pétris de culture grecque antique se sont rendus à plusieurs reprises en Grèce avant d'écrire leurs récits de voyage : de 1947 à 1966 pour l'écrivain français, en 1964, 1973 et 1975 pour l'écrivain polonais. S'ils voyagent aux mêmes époques dans un même lieu qui a toujours nourri leur imaginaire et structuré leur pensée, leurs origines et leurs histoires personnelles sont sensiblement différentes : au sortir de la Seconde Guerre mondiale, Lacarrière se rend en Grèce à 22 ans dans le cadre d'un voyage organisé en collaboration avec le pays d'accueil, avant d'y mener des voyages plus solitaires et sans limite particulière de temps. Herbert, lui, se rend en Grèce à un âge plus avancé - quarante ans - après un parcours semé d'embuches, dans le climat dictatorial de la Pologne communiste. Herbert se sent donc en sursis lorsqu'il quitte la Pologne: il exprime à plusieurs reprises sa crainte de ne jamais pouvoir revenir en Grèce, et la culpabilité d'avoir eu la chance de partir quand ses compatriotes souffrent dans son pays. Ces vécus différents colorent évidemment le regard porté sur la Grèce et le discours qui en découle. Lecteurs avertis des écrivains-voyageurs qui les ont précédés en Grèce, les deux auteurs se situent eux-mêmes par rapport à cette tradition et s'interrogent sur la singularité de leur expérience. L'avant et l'après découverte des lieux sont constamment mis en relation, sur un mode très souvent poétique. Il faudra donc se demander dans quelle mesure la dialectique du réel et de l'imaginaire structure l'écriture $d u$ voyage. Le voyage opère-t-il comme simple révélateur du réel, ou aussi comme vecteur d'un nouvel imaginaire des lieux ancré dans le réel comme dans la subjectivité du souvenir et de la perception des lieux ? On pourra alors cerner les contours de la Grèce recherchée par les auteurs. En effet, leurs textes donnent à voir un espace de référence précis - l'entité géographique Grèce, telle qu'elle existe au moment de leur voyage - à travers une " multifocalisation des regards ", pour reprendre l'expression de Bertrand Westphal (Westphal, 2007 : 188). Or la cartographie des voyages entrepris par Lacarrière et Herbert et le récit qu'ils en font témoignent d'une approche nécessairement sélective de la Grèce. L'Antiquité grecque, sans surprise, y est abondamment évoquée. Derrière ces choix se dessine une représentation politique de la Grèce et de sa place en Europe. Ce travail voudrait prendre la suite d'études aussi bien françaises que polonaises et grecques sur le philhellénisme romantique et ses prolongements au xx siècle (Basch, 1991, 1995; Borowska, 2007, 2012) en adoptant une approche comparatiste ouverte sur l'Est européen. En effet, le second vingtième siècle y est relativement peu étudié, ou alors présenté comme un épilogue du mouvement né au XIX siècle. Pourtant, la guerre froide qui partage l'Europe ainsi que le vaste mouvement de décolonisation et de remise en cause de la suprématie occidentale (Gély, 2012) qui s'enclenche au moment où Lacarrière et Herbert se rendent en Grèce réoriente en partie leur représentation des lieux. Loin d'être anodin dans une Pologne sous domination soviétique, l'intérêt pour la culture grecque peut sonner comme une revendication d'appartenance à l'héritage classique de l'Europe occidentale. Comment donc, dans L'Eté grec et Le Labyrinthe au bord de la mer, se construit, se fissure et se recompose par le voyage l'imaginaire de la Grèce? 


\section{Aux sources de l'invitation au voyage en Grèce : l'imaginaire des livres}

\subsection{Un imaginaire nourri par l'enseignement scolaire, les lectures et le rejet du présent}

2 L'attente du voyage naît d'un goût pour la culture grecque découverte par le prisme de l'Antiquité dès l'enfance, pour Herbert comme pour Lacarrière (étude des civilisations antiques au collège, apprentissage du grec à partir du lycée, licence de lettres classiques). L'auteur de L'Eté grec explique que la lecture des tragiques grecs avait le voyage en Grèce pour horizon constant, un horizon semi-réel, semi-merveilleux : « des syllabes magiques et vibrantes derrière lesquelles se profilait une Grèce rêvée et par la suite rencontrée. » (Lacarrière, 1984 : 157). Le voyage apparaît comme le complément indispensable à la connaissance de la Grèce par les livres : «Il n'est pas pour moi de culture autre que vécue » écrit l'écrivain français (Lacarrière, 1984 : 157). L'imaginaire $\mathrm{du}$ voyage est également nourri par les lectures des grands récits de voyage en Grèce qui valorisent souvent l'Antiquité au détriment de l'histoire plus récente du pays, négligée sinon ouvertement méprisée (Basch, 1995). Herbert, lui, a étudié la culture antique à l'école ainsi que le latin. Il approfondit sa connaissance de la culture grecque antique au cours de ses études par intérêt personnel. Dans sa riche biographie consacrée au poète polonais, Brigitte Gautier détaille la chronologie des lectures de Herbert (Gautier, 2018: 54, 125) : Hésiode, Pindare, Solon, Euripide, Aristote, puis les grands écrivains voyageurs, d'Hérodote à Chateaubriand. Lorsqu'il dépeint la Grèce et tente de remonter le temps jusqu'à l'Antiquité, Herbert a conscience des chants de ses prédécesseurs (les «fantômes" de Marguerite Yourcenar) et les convoque même à de nombreuses reprises. Les épigraphes des chapitres du Labyrinthe témoignent de la perspective antique adoptée par l'auteur : quatre sur dix sont issues d'auteurs antiques. Ces citations, ajoutées à celles qui ponctuent le texte, témoignent de l'érudition de Herbert qui se documente avant et après le voyage. Enfin, au-delà de l'école et des lectures personnelles, le rêve du voyage en Grèce se trouve renforcé par l'insatisfaction ressentie face à la réalité terne dans laquelle, toutes proportions gardées, les deux auteurs évoluent. La Grèce apparaît alors comme un ailleurs merveilleux, inatteignable. Lacarrière écrit ainsi « Découvrir les mythes grecs à cet âge, c'est (...) pressentir, audelà des murs gris des lycées, l'existence d'une terre d'azur et de sang - la Grèce - que je rêvais intensément de rencontrer." (Lacarrière, 1984: 14). La palette de couleurs évoquée par l'auteur illustre le contraste moteur entre la réalité présente et l'image de l'ailleurs: au gris s'opposent l'azur et le rouge tranchants d'une Grèce à la fois apollinienne et dionysiaque. A la métaphore de l'enfermement se substitue une formule onirique dans laquelle le lieu évoqué par le terme vague de " terre » n'est défini que par deux couleurs, signe que ce pays est associé à une vaporeuse image de liberté et de sensualité. Le contraste entre l'ici et l'ailleurs est encore plus saisissant chez Herbert, originaire d'un pays qui se trouve alors sous la tutelle de l'URSS. S'il a réussi à quitter la Pologne en juillet 1963 et a pu voyager en Europe avant de s'embarquer en Grèce au mois d'août 1964, c'est au prix de nombreuses tentatives infructueuses. A chaque nouveau départ, Herbert est confronté à des difficultés administratives concernant son passeport qui est utilisé comme un instrument de chantage par la police politique qui commence alors à s'intéresser à lui (Gautier, 2018: 120). Dans le chapitre intitulé 
"L'Acropole », Herbert revient sur ce long parcours qui a précédé le voyage en Grèce et qui l'a rendu ainsi plus désirable encore :

Trzeba bylo pokonać przeszkody, mury urzĘdów, zamkniĘte drzwi, korytarze i całĄ maş̨ rozmienionych na drobne cerberów za biurkami, wahajĄcych sie czy przybić magicznĄ pieczĘć, trzymajĄcych, plątajĄcych w palcach nitk̨̨ mojej przygody. (Herbert, $2000: 129$ )

Il avait fallu vaincre les obstacles, les murs des administrations, les portes fermées, les corridors et toute une foule de cerbères derrière des bureaux, hésitant à apposer le tampon magique, tenant et enroulant entre leurs doigts le fil de mon aventure. (Herbert, 2015 : 168)

3 L'accumulation d'obstacles, rendue ici par la longue énumération d'opposants sans visage, permet de comprendre par contraste l'envie irrépressible de voyage et de liberté éprouvée par l'auteur. L'imaginaire de la Grèce et du voyage en Grèce se trouve ainsi entretenu par un rejet - plus ou moins fondamental - de l'environnement présent de l'auteur, qui fait de la Grèce un idéal encore plus inaccessible.

\subsection{L'imaginaire avant le voyage : une Grèce figée}

L'attente du voyage et de la découverte du pays tant admiré et étudié s'avère propice à la formation d'un réseau d'images intuitives et de connaissances scolaires ou livresques. L'Eté grec s'ouvre sur une description d'un imaginaire modelé par l'apprentissage scolaire :

Ces images étaient celles d'une terre de ruines, de colonnes, de frontons écroulés, de tombeaux éventrés au milieu de l'humus des forêts. Des humains meublaient parfois ces ruines mais ils avaient la fixité du marbre; drapés dans de blanches tuniques, ils regardaient la mer ou le ciel, en poses hiératiques. (Lacarrière, 1984:

14)

5 Temples en ruine, marbre, tuniques blanches: on retrouve ici l'influence du philhellénisme romantique et les stéréotypes qui l'accompagnent. Evincés les âges qui succèdent à l'Antiquité, accessoires les hommes qui peuplent ce pays, effacées les couleurs chatoyantes qui ornaient les monuments antiques (Jockey, 2015). Par l'accumulation d'éléments de décors juxtaposés sans interaction et par le choix de l'imparfait, Lacarrière rend sensible la fixité marmoréenne de son imaginaire, nourrie par les représentations picturales de la Grèce telles que les gravures accompagnant $L e$ Voyage du jeune Anacharsis en Grèce de l'Abbé Barthélémy ou les peintures d'Hubert Robert. Ces images ne demandent qu'à être animées et c'est précisément le voyage qui est perçu comme le moyen de donner vie à toutes ces représentations mentales figées, ou de les éliminer si elles s'avèrent fallacieuses, comme le suggère la fin de la citation. Si Herbert s'étend moins que Lacarrière sur l'imaginaire de la Grèce qui fut le sien dans l'enfance, il livre néanmoins un certain nombre d'anecdotes et de réflexions sur sa façon de se représenter a priori le pays qu'il n'avait pas encore visité. Dans Le Labyrinthe, cette question sert d'entrée en matière au chapitre intitulé « L'Acropole » :

Nie ma na świecie budowli, która tak trwale okupowała moją wyobraźniĘ. ZdiĘcia, rysunki, opisy stanowiły pokarm wodnisty, pozbawiony zapachu, kolory i tła. Znałem nieźle topografiĘ, wymiary i kontur głównych świątyń, ale cały ich zespół położony był nieodmiennie na płaszczyźnie, miał barwę gipsu, nie oddychał światłem, a niebo nad nim było z papieru. (Herbert, 2000 : 95)

Aucun autre édifice n'occupa autant mon imagination. Les photos, les dessins, les descriptions étaient un aliment liquide, dépourvu d'odeur, de couleur, de profondeur. Je connaissais plutôt bien la topographie, les dimensions et les 
contours des principaux temples. Leur ensemble pourtant était couleur de chaux, posé immuablement sur une surface plane, il ne respirait pas la lumière, et le ciel au-dessus était de papier. (Herbert, 2015 : 125)

On retrouve dans cette description l'absence de vie évoquée par Lacarrière. L'Acropole y semble un décor de carton-pâte en deux dimensions, même s'il s'appuie sur de solides connaissances théoriques. De ces représentations aseptisées naît précisément l'envie du voyage.

\title{
1.3. La perspective du voyage : entre enthousiasme et appréhension
}

7 Avant même d'arriver en Grèce, Herbert et Lacarrière s'interrogent sur les enjeux que revêt pour eux le voyage. Il s'agit d'abord de rectifier l'imaginaire scolaire afin de parvenir à une représentation plus authentique des lieux. Sans concession, Lacarrière le décrit comme traversé par un « cortège d'images trompeuses, conventionnelles mais incroyablement tenaces, puisque pour beaucoup, elles continuent de signifier la Grèce » (Lacarrière, 1984 : 14). Herbert quant à lui affirme son besoin presque vital de se confronter à la réalité par le voyage à travers un lexique empreint de sensualité :

\begin{abstract}
SkĄd wiĘc ta przemożna wola konfrontacji, ta pasja pchająca do zbliżenia fizycznego, pożĄdanie, aby położyć rĘce, zjednoczyć siĘ cieleśnie, a potem oderwać się, odejść i unieść ze sobĄ co - Obraz? Dreszcz ? (Herbert, 2000: 129)

D'où me venait donc cette volonté farouche de confronter, cette passion incitant au rapprochement physique, ce désir de poser mes mains, de m'unir charnellement, puis de m'en arracher, de partir et d'emporter - quoi ? Une image? Un frisson? (Herbert, $2015:$ 168)
\end{abstract}

Passer du schéma au «frisson " grâce à l'expérience vécue, voilà tout l'enjeu du voyage, pour Herbert. A côté de l'enthousiasme pour le voyage, émergent aussi parfois des craintes, notamment celle de la déception face à la confrontation du réel et de l'imaginaire. Herbert s'en explique dans un passage de "L'Acropole » :

Kiedy jechałem tam, rósł we mnie strach, że konfrontacja zniszczy to, co przez wiele lat budowały cierpliwe domysły. I czy bĘdĘ miał odwagĘ przyznać siĘ (choćby przed samym sobĄ) (...)? Czy wezmĘ udział w trwającym od wieków sprzysį̨żeniu zachwytu (...). (Herbert, 2000: 95)

Alors que je me rendais là-bas, la peur me gagnait de voir la confrontation détruire ce que mes patientes suppositions avaient édifié pendant des années. Et aurais-je le courage de le reconnaître (ne serait-ce que devant moi-même) (...) ? Allais-je entrer dans la confrérie séculaire du ravissement (...). (Herbert, 2015 : 125)

9 La succession d'interrogatives et d'hypothétiques vient ici suspendre le récit avant le basculement de la confrontation au réel et donne à entendre l'appréhension de l'auteur. La perspective du voyage en Grèce a tellement soutenu Herbert dans les heures sombres que la désillusion serait pour lui tragique. Conscient de s'inscrire dans une succession d'illustres visiteurs, il craint aussi de mimer l'émotion des autres, mise à distance avec ironie, plutôt que de la ressentir personnellement. Lacarrière et Herbert mènent donc chacun une forme d'examen de conscience préalable qui retarde le récit de voyage et renforce du même coup les attentes qui l'accompagnent. 


\title{
2. L'imaginaire face à la réalité du voyage : émotion et reconfigurations
}

10 La confrontation entre l'imaginaire et le réel passe par l'un des enjeux essentiels du voyage : celui d'un face à face intime et charnel dans lequel les connaissances mêmes ne doivent pas interférer, du moins dans un premier temps. Lacarrière explique cette attitude à l'occasion de sa visite de Cnossos :

Et je me sentais nu, en ce lieu presque nu : pas d'histoire à remémorer ni d'archéologie à retrouver, rien que ce contact immédiat avec un site qui me parlait chaque jour par ce qu'il avait de moins profond et pourtant de plus révélateur : le silence et la solitude de Cnossos en septembre (Lacarrière, 1984 : 119)

11 Avant de décrypter l'histoire du site, l'auteur prend le temps de ressentir l'atmosphère du lieu, dans sa réalité la plus quotidienne, sans aucune médiation comme le souligne l'image de la nudité. Confrontée à la réalité grâce à l'expérience physique, l'image intérieure se fissure parfois, se recompose et s'enrichit.

\subsection{Fissures de l'image : choc de la prise de conscience d'un continuum historique, sensation d'étrangeté}

12 Les réactions contrastées qui découlent de la confrontation oscillent entre concordance, remise en cause et découverte inattendue. La confrontation la plus marquante et la plus déroutante pour Lacarrière semble être celle de Delphes. L'écrivain s'y rend en 1947 alors que la ville est occupée par les insurgés communistes, dans un pays en pleine guerre civile. L'auteur y prend subitement conscience de l'histoire tragique que la Grèce traverse alors :

\begin{abstract}
Ainsi, toute l'après-midi je pus errer seul dans les ruines. Silence. Solitude. Pas un seul bruit vivant, si ce n'est par moment le cri des gypaètes (...) Delphes était vide, abandonné, livré à tous les fantômes de l'histoire. (...) Ce jour-là, dans cette nuit de Delphes et ce silence des montagnes où nous épiaient, sans aucun doute, les partisans, je sentis qu'une Grèce mourait en moi et qu'une autre naissait. (...) Je dois à ce premier séjour à Delphes, à cette rencontre avec la guerre, de m'avoir délivré à jamais du mirage des pierres (Lacarrière, 1984 : 16-17)
\end{abstract}

13 L'image d'une Grèce figée dans une sorte de hors-temps se fissure ici, comme le souligne le passage de l'imparfait au passé simple qui isole le moment de la révélation ( « je sentis »). Derrière le "mirage des pierres » véhiculé par les livres se manifeste la pérennité de la Grèce au-delà d'une époque idéalisée dans laquelle l'Occident voudrait la figer. Pour Herbert, le choc survient face à la découverte de l'Acropole. Dans le chapitre qui lui est consacré, l'auteur commence par exprimer sa difficulté à adhérer à l'admiration automatique dictée par le discours scolaire à l'égard de tout ce qui est antique :

Czym jest Akropol jako całość? Odpowiedź w podrĘczników szkolnych brzmiałaby: harmonijnym zespołem architektury. Ale nieuprzedzone spojrzenie odkrywa, że tak nie jest. (Herbert, $2000: 107)$

Qu'est-ce que l'Acropole en tant qu'ensemble? La réponse des manuels scolaires serait : un ensemble architectural harmonieux. Mais ce n'est pas le cas pour un regard innocent. (Herbert, $2015: 140$ ) 
Par le jeu d'adjectifs qualificatifs antithétiques, l'ensemble architectural est dépeint comme un enchevêtrement de monuments aux proportions inégales qui semble peu compatible avec la définition scolaire imaginée par Herbert. Plus loin, l'auteur écrit :

Była już noc. Wokół wzgórza prawie pusto. Jeśli dobrze przypominam sobie tĘ chwilę, zaczĄłem liczyć, po prostu liczyć kolumny - osiem, siedemnaście i znów osiem, siedemnaście. (Herbert, $2000: 130)$

Il faisait déjà nuit. Il n'y avait presque personne autour de la colline. Si je me rappelle bien cet instant, je commençais à compter, compter tout simplement les colonnes : huit, dix-sept et à nouveau huit, dix-sept. (Herbert, 2015 : 169)

Récurrente dans les récits de découverte d'un lieu longtemps imaginé, on retrouve la solitude du voyageur face au monument qui était celle de Lacarrière au moment de sa prise de conscience de Delphes. L'admiration ne s'empare pas d'emblée du poète polonais. Par son regard comptable, par les chiffres donnés en toutes lettres, il tient l'Acropole à distance. L'auteur se sent en décalage avec l'admiration sans borne qui était notamment celle d'Ernest Renan : "Je ne pouvais répéter ni l'émotion ni la prière d'un humaniste du siècle passé. Les paroles de foi rationnelle n'arrivaient pas à traverser ma gorge » (Herbert, 2015 : 170 - Przez gardło nie mogły mi przejść słowa racjonalistycznej wiary, Herbert, $2000: 131)$. Déroutante, la rencontre entre l'Acropole imaginaire et l'Acropole réelle n'en est pas moins source d'émotion. Après avoir cité la Prière sur l'Acropole dans laquelle il ne se reconnaît pas, Herbert livre sa propre perception des lieux, empreinte d'humilité et d'émotion simple, qui incorpore pleinement au substrat antique toute l'histoire de la Grèce qui se trouve comme imprimée sur la colline :

Akropol, jaki miałem przed oczami, sprowadzony do skieletu, odarty z ciała, był dla mnie zarówno dziełem woli, ładu, jak i chaosu, artystów i historii, Peryklesa i Morosiniego, Iktinosa i grabieżców. I dotykajĄc wzrokiem jego ran i okaleczeń, doznawałem uczuciam y którym podziw mieszał sį̨ z litością. (Herbert, 2000 : 131) Sous mes yeux, l'Acropole réduite à un squelette, dépouillée de sa chair, était à la fois l'œuvre de la volonté, de l'ordre et du chaos, des artistes et de l'histoire, de Périclès et de Morosini, d'Ictinos et des pillards. Et effleurant du regard ses blessures et ses mutilations, j'éprouvais une admiration mêlée de pitié. (Herbert, $2015: 170)$

16 Loin de figer l'Acropole dans une majesté antique inatteignable, Herbert en fait un portrait attendri qui souligne sa vulnérabilité et son rôle de témoin des autres phases de l'histoire de la Grèce. Autre confrontation déroutante de l'imaginaire au réel, commune aux deux écrivains : la Crète. Lacarrière, qui s'y rend en septembre 1950, y est subitement confronté à l'idée que la Grèce n'est pas la patrie spirituelle qu'il s'imaginait :

Et ce soir-là, devant cette langue presque inconnue, j'eus le sentiment que la Grèce et la Crète plus encore - avaient finalement peu à voir avec l'Occident. Ce voyage vers la mère-patrie de nos concepts, aux racines de notre raison, à la source claire de nos rêves et de nos mythes me révélait un aïeul méconnaissable, parlant une langue si déconcertante par ses sons, si orientale par ses gestes que je me sentis dérouté. (Lacarrière, $1984: 129$ )

La filiation que l'auteur croyait établie est tout à coup mise en doute, du fait de la langue qu'il ne comprend pas et qu'il apprendra par la suite, elle pousse le voyageur à découvrir une Grèce plus orientale. Cette prise de conscience est fréquente dans les récits de voyage en Orient du siècle qui précède, mais elle s'accompagne souvent d'un mépris pour ce qui est perçu comme une décadence par rapport à l'Antiquité (Basch, 
1991 : 24). La Crète s'avère être également le lieu d'une expérience troublante pour Herbert, alors même que l'apparition spectaculaire de celle-ci laissait présager un émerveillement sans ombre : «C'est ainsi que la Crète a commencé pour moi, venant du ciel, comme une divinité.» (Herbert, 2015 : 15 - Tak się zaczĘła dla mnie Kreta, od nieba, jak boskowo Herbert, 2000 : 8). Il ressent une profonde déception face à la découverte des fresques de Cnossos, qui jouissaient pourtant dans son imaginaire d'une aura de chef-d'œuvre en attente d'être contemplé. Il rend palpable l'accélération du temps et met ainsi en scène le moment du face à face : l'attente de longues années se termine en course vers l'objet rêvé. « Je traversai au pas de course (...) les salles du rezde-chaussée (...) je montai au premier étage avec un battement de cœur » (Herbert, 2015 : 16 - przebiegłem (...) sale na parterze (...) i w biciem serca wszedłem na piętro pierwsze Herbert, 2000 : 9). Résultat: «Et puis quoi? Rien. Je regardai hébété Les Dauphins, (...) et Le Prince aux lys, sans émotion ni sympathie. » (Herbert, 2015 : 16 - I co? Nic. Bez wzruszenia i bez sympatii patrzyłem tĘpo na Delfiny (...) i na Księcia wśrĄd lilii. Herbert, 2000: 10). La syntaxe, faite de courtes phrases juxtaposées et peu soignées, accentue la retombée de l'enthousiasme. Herbert finit néanmoins par identifier la raison de sa déception : le découvreur de Cnossos, Sir Arthur Evans, a fait remplacer les peintures originales par des reproductions, compromettant ainsi la possibilité d'un contact direct et sans médiation avec les chefs-d'œuvre. L'imaginaire du voyage forgé par les deux écrivains se trouve ainsi bousculé plusieurs fois, ce qui les amène à le réinterroger ou à le rectifier.

\subsection{L'expérience de la concordance imaginaire-réel}

Dans d'autres cas, lieu imaginaire et lieu réel concordent. Cette harmonie est souvent célébrée sur un mode poétique marqué par une compénétration entre passé mythique et présent de l'écriture. Les descriptions de la découverte de Mycènes par les deux écrivains sont unies par de nombreuses convergences. Parmi elles, l'idée d'un lieu marqué à jamais par le crime, encore hanté par les Atrides. A propos de « cette pérennité fantomatique " Lacarrière écrit ainsi : "Ils continuent manifestement de régner, de chasser, d'ordonner quelque part, entre le monde des ombres et celui des vivants » (Lacarrière, $1984 ; 170)$. On retrouve cette impression chez Herbert qui décrit la demeure des Atrides en ces termes:

Pałac jest raczej posĘpnĄ fortecĄ. Została tylko krew i kamienie. Kiedy szosa skrĘca nagle, ukazuje si巨̨ oczom grecka stolica zbrodni. (...) Mykeny wzwierajĄ nieodparte wrażenie łiejsca nayiedzonego tragediĄ. (Herbert, $2000: 65$ )

Le palais est plutôt une forteresse lugubre. Seul le sang et les pierres ont subsisté. A un brusque tournant de la chaussée apparaît la capitale grecque du crime. (...) Mycènes produit cette impression irrésistible de lieu visité par la tragédie.» (Herbert, $2015: 89$ )

La Mycènes réelle semble ici se fondre avec l'imaginaire des deux écrivains pour lesquels elle est associée à la famille maudite, nourris qu'ils sont de la lecture des tragiques grecs. Autre point commun entre les deux auteurs : le caractère lugubre de la ville se reflète dans le paysage qui l'entoure. Lacarrière compare la ville à un « repaire d'aigles » dans lequel il fallait lutter pour vivre :

Rien de plus tranchant (exacerbé encore par les contrastes forts du paysage) que ce repaire où s'entassaient or et diadèmes, coupes et tapis de pourpre, face à ce lieu dénudé et brûlé où l'eau elle-même se cache très loin sous la terre. (Lacarrière, $1984: 172)$ 
eléments eux-mêmes semblent prendre peur face à Mycènes. La nature alentour, « dénudé[e] » et «brûlé[e] », est comme anéantie par les crimes qui s'y sont déroulés. On peut également remarquer un contraste visuel entre l'or et la couleur rouge associée aux tapis de pourpre mais aussi au sang. Ce contraste des couleurs se retrouve dans l'extrait de l'Iliade que Herbert a choisi de citer en début de section: "Voici Mycènes pleine d'or et le palais sanglant des Pélopides.» (VII, 180, XI, 4, 1992). Ce passage sert vraisemblablement d'intertexte à Herbert (et peut-être aussi à Lacarrière) lorsqu'il écrit plus loin: «La nuit descend des montagnes, mais le nid des Atrides scintille encore d'une teinte fauve de vieil or et de sang ancien.» (Herbert, 2015 : 91 Noc zchodzi z gór, ale gniazdo Atrydów świeci jeszcze rudnym kolorem starego złota starej krwi Herbert, 2000 : 66). On retrouve ici l'association chromatique de l'or et du rouge sang, ainsi que la métaphore du «nid » présente chez Lacarrière, qui associe les Atrides à des rapaces. Le paysage alentour est dépeint comme «sauvage, fermé. Le ciel, effrangé par les crêtes des montagnes est suspendu au-dessus de Mycènes comme un nuage pétrifié de malheur.» (Herbert, 2015: 89 Pejzaż jest dziki, zamkniĘty. PostrzĘpione wierzchołkami gór niebo wisi nad Mykenami zakrzepła chmurĄ nieszczĘścia. Herbert, 2000: 65). La réalité du lieu semble ainsi se confondre avec l'image intérieure que l'on s'en faisait: une terre aussi fascinante qu'inhospitalière. Dernier point commun entre les deux récits : l'attention portée à la survivance du lieu. Herbert exprime son admiration pour les «murs cyclopéens", "chef-d'œuvre de construction puisque pendant trois mille ans et demi, ils ont résisté aux avalanches de l'histoire.» (Herbert, 2015 : 90 - cyklopowe mury sĄ arcydziełem konstrukcji, skoro przez trzy i pół tysiąca lat opierały się nawałnicom historii. Herbert, $2000: 65)$. Chez Lacarrière, cette réflexion passe moins par la pierre que par la continuité de la représentation des lieux à travers les âges : «d'Eschyle jusqu'à Séféris, c'est un même sillage, un même flot d'images intensément semblables qu'engendre le seul mot de Mycènes » (Lacarrière, 1984 : 173). Certaines étapes du voyage en Grèce enrichissent donc l'imaginaire sans le détruire. Le réel vient incarner ce que l'esprit se représentait, l'associer à des sensations et des couleurs imperceptibles jusqu'alors physiquement. Mythe et réalité s'interpénètrent sans s'exclure, vue et vision s'entremêlent. D'autres lieux permettent cette concordance entre image et réalité. Ces expériences marquantes permettent ainsi de dégager un certain nombre de constantes dans le récit de la confrontation entre l'imaginaire et le réel : le moment de l'apparition, souvent mis en scène par un effet d'attente travaillé, l'éveil des sens ou "polysensorialité " pour reprendre un concept de géocritique, ainsi que la solitude face au monument comme occasion d'une révélation privilégiée.

\section{Le récit de voyage comme témoignage : quel discours sur la Grèce?}

Les récits de Lacarrière et Herbert peuvent aussi se lire dans une perspective plus politique. La Grèce permet à l'un de témoigner indirectement des privations de liberté subies en Pologne, à l'autre du regard lacunaire de l'Europe occidentale sur la culture grecque.

Carnets, Deuxième série - 20 | 2020 


\subsection{Un témoignage hommage : le cas du Labyrinthe au bord de la mer}

Les essais du Labyrinthe sont traversés de passages dans lesquels l'auteur exprime à la fois sa gratitude d'avoir pu se rendre en Grèce et sa compassion pour ses proches qui n'en ont pas eu la chance. Le passage qui le montre le plus clairement se situe dans l'essai intitulé La petite âme :

No więc tak: stojĄc na Akropolu przywoływałem dusze moich poległych kolegów, użalałem sį̨ nad ich losem, już nawet nie nad śmierciĄ okrutnĄ, ale współczułem, że odebrana im została niewyczerpana wspaniałość świata. Sypałem ziarna maku na zapomniane groby.

(...) Skoro zostałem wybrany (...) to muszĘ temu wyborowi nadać sens, odebrać mu jego przypadkowość i dowolność.

(...) Wyobrazić sobie, że jestem delegatem czy posłem tych wszystkich, którym się nie udało. (...) I żebym to, co z nich pojąłem, potrafił przekazać innym. (Herbert, $2000: 90)$

Alors que je me trouvais sur l'Acropole, j'invoquais les âmes de mes camarades morts, je déplorais leur sort, non tant leur mort cruelle que le fait que la splendeur inépuisable du monde leur ait été ôtée. Je répandais des graines de pavot sur leurs tombes oubliées.

(...) Puisque j'ai été choisi (...), je dois donner un sens à ce choix, lui ôter son caractère contingent et arbitraire.

(...) M'imaginer que je suis le délégué ou le député de tous ceux qui n'ont pas survécu. (...) Pour que je sois capable de transmettre à d'autres ce que j'en ai compris. (Herbert, 2015 : 121)

Herbert évoque avec pudeur le sort de ses compatriotes, dans une prière qui rappelle celle de Renan, mais qui au lieu de s'extasier une fois de plus sur la beauté des lieux, pleure sur ceux qui n'ont pu la contempler. Cette inversion explique l'une des raisons d'être du recueil d'essais : celui du témoignage. Ce témoignage se veut le plus objectif possible, afin de ne pas dénaturer les lieux et rendre possible leur appropriation par d'autres. On comprend dès lors l'obstination avec laquelle l'auteur s'attache à décrire les chefs-d'œuvre qu'il lui est donné de voir et à en proposer une somme des connaissances. La voracité qui est celle de l'auteur dans son rapport aux œuvres d'arts et à la connaissance s'explique elle aussi par ce rôle de « délégué ». Chaque essai, dédié à un proche, constitue ainsi une petite victoire sur le temps, qui ne lui offrira peut-être pas la possibilité d'un retour. En dehors de ces présents d'encre et de papier, Herbert se montre également solidaire de ses proches en dressant de subtils parallèles entre les cités dont il observe les ruines en Grèce, et les pays d'Europe de l'Est sous domination soviétique. Ainsi du chapitre intitulé «L'affaire de Samos », dans lequel l'auteur prend le parti inattendu de Samos, opprimée par Athènes, et remet ainsi en cause l'objectivité du regard traditionnellement porté sur Athènes, biaisé par des sources antiques athénocentrées. Il est d'ailleurs intéressant de noter que cet essai est dédié au poète Miroslav Holub, traducteur de Herbert licencié de son travail au lendemain de l'invasion de la Tchécoslovaquie par les troupes du pacte de Varsovie. En plus de cette dédicace provocatrice (supprimée par la censure polonaise, comme l'explique Brigitte Gautier dans l'apparat critique du Labyrinthe, 2015 : 272) l'essai est ponctué de sousentendus invitant à voir derrière le drame de Samos, celui de l'Europe de l'Est. L'auteur conclut par exemple un paragraphe consacré à la ligue de Délos, véritable instrument de domination entre les mains d'Athènes, par le constat discret qui suit: "L'histoire antique n'est pas la seule à connaître ce genre de situations. », avant d'en revenir à 
l'Antiquité. Le chapitre se clôt ensuite sur une phrase qui sonne comme la chute d'un exemplum historique :

Jaki wiĘc pożytek może mieć przypomnienie tej odległej afery (...)? MyślE, że ten: najeźdźcy wracając $\mathrm{z}$ wojny przynoszą $\mathrm{w}$ fałdach mundurówm na podeszwach butów - zarazek, od którego chorować bĘdzie ich własne społeczeństwo, ich własne swobody. (Herbert, 2000 : 144)

Quel profit y a-t-il donc à rappeler cette affaire ancienne (...) ? Ceci selon moi : les envahisseurs qui rentrent de la guerre rapportent dans les plis de leurs uniformes, sur les semelles de leurs souliers, le germe qui infectera leur propre société, leurs propres libertés. (Herbert, $2015:$ 185)

Cette dernière phrase au présent de vérité générale se distingue par son absence d'ancrage historique. Au terme du chapitre s'opère ainsi un renversement : Samos, qui était au cœur de l'essai apparaît finalement comme un simple exemplum historique grâce auquel, à la manière de Plutarque, Herbert peut transmettre avec discrétion, pédagogie et force de persuasion une morale, ou en l'occurrence un avertissement.

\subsection{Le discours de la grécité}

On retrouve dans l'œuvre de Lacarrière l'enjeu du témoignage, mais elle n'est pas dictée par les mêmes raisons que chez Herbert. La rédaction et la publication de l'Eté grec en 1976 est présentée par l'auteur lui-même comme une tentative de soutien à la Grèce au moment où elle vit les heures sombres de la dictature des colonels. Il s'agit de faire connaître la Grèce dans toute sa richesse, en se défaisant du prisme antique et en s'ouvrant à toutes les autres facettes de la Grèce trop souvent méprisées par l'Occident, et parfois reniées par les Grecs eux-mêmes (Tziovas, 2014). Lacarrière ainsi que Herbert mais dans une moindre mesure, plaident pour la prise en compte des autres aspects de l'histoire de la Grèce, découverts au fil d'un voyage qui le fait naviguer d'une époque à l'autre. Ainsi de la période byzantine, découverte lors de son voyage au mont Athos : " J'y découvrais une Grèce ignorée, reléguée dans les manuels d'histoire de l'Occident à la fin des chapitres ou dans les notes en bas de page : la Grèce byzantine. " (Lacarrière, 1984 : 66). C'est pourquoi cette découverte ne donne pas lieu à la description traditionnelle de la confrontation entre imaginaire et réalité :

Ce mont d'Athos alors n'évoquait pour moi qu'une image : celle de ce monastère aérien, suspendu entre ciel et terre, zébré de passerelles. Je ne savais rien de cette montagne sainte, de son histoire, de ses légendes. A l'encontre de Mycènes ou de Delphes, elle était pour moi un monde vierge, inconnu, un monde que je n'attendais pas. (Lacarrière, 1984 : 66-67)

Ce n'est pas par hasard si Lacarrière place le chapitre consacré au mont Athos au début de son œuvre, avant le récit de la rencontre avec les sites antiques. Il s'agit pour lui de mettre l'accent sur ce qu'il a découvert être la véritable essence de la Grèce: sa continuité historique exceptionnelle qui fait d'elle ce que les géographes appellent un "peuple monde de la longue durée " (Bureau, 2001). Cette longévité de la Grèce est résumée par cette belle formule de Herbert, au tout début du Labyrinthe : un "pays où les horloges égrènent les millénaires » (Herbert, 2015 : 13). Les deux écrivains prennent soin d'intégrer toutes les périodes de la Grèce dans leurs écrits, même si elles sont parfois inféodées à la volonté de décrire un monument antique chez Herbert. Le vecteur principal de cette continuité réside pour Lacarrière qui rejoint par là tous les poètes grecs de son temps, dans la pérennité de la langue grecque: "La Grèce d'autrefois, morte en ses temples et ses pierres, vit toujours sur les lèvres des enfants 
grecs. » (Lacarrière, 1984 : 224). Il raconte avoir pris la mesure de cette continuité linguistique en entendant un enfant dire d'un crabe mourant sur la plage «Charopalévi »-« Il lutte avec Charon ». Ce verbe qui signifie mourir est forgé de deux termes qui existaient déjà dans l'Antiquité. Reprenant à dessein l'idée de miracle, galvaudée à ses yeux par le philhellénisme occidental, Lacarrière explique que ces termes expriment exactement ce qu'est le «miracle grec - plus vrai, plus important peut-être que celui des siècles classiques " (Lacarrière, 1984 : 282). Non sans humour, Herbert dénonce lui aussi le caractère lacunaire et sclérosant de l'imaginaire traditionnel qui néglige la réalité vivante de la Grèce : « Il me semble que la conclusion à tirer de cette anecdote est que les nations ont mieux à faire que de ressembler à l'idéal imaginé par des humanistes romantiques.» (Herbert, 2015 : 14 - MyślĘ, że z anegdoty tej wysnuć należy jeden wniosek: narody majĄ ważniejsze, bardziej elementarne sprawy niż troskĘ, by upodobnić sį̨ do ideału wymyślonego przez romantycznych humanistów. Herbert, $2000: 8$ ).

Ainsi, Lacarrière et Herbert retracent avec un esprit critique relativement nouveau dans le genre du récit de voyage en Grèce leurs émotions et réflexions qui naissent de leur découverte attentive des lieux. Sans jamais se départir d'un regard profondément poétique, l'un et l'autre s'emploient en effet à rectifier les biais de l'imaginaire et des récits de voyage en Grèce. Cela passe, chez Lacarrière, par une remise en cause de la focale antique et la célébration de la Grèce de l'ombre : description de l'art de vivre des Grecs qu'il rencontre, des lieux situés en dehors des circuits touristiques de l'époque, de l'histoire de la Grèce après l'Antiquité, de la langue grecque moderne. Chez Herbert, les lieux visités restent assez traditionnels, pressé qu'il est de se mesurer aux chefsd'œuvre tant de fois imaginés avant de se retrouver « au cap sombre du temps ». Mais cela n'empêche pourtant pas l'auteur de remettre en cause les idées reçues sur la Grèce. Avec discrétion, le poète renverse souvent la perspective sur l'objet ou le sujet qu'il évoque afin d'en montrer ce que ses prédécesseurs ont pu laisser dans l'ombre. De ces récits marqués par une histoire européenne mouvementée, émergent une pratique du voyage et un imaginaire de la Grèce renouvelés, amendés et élargis.

\section{BIBLIOGRAPHIE}

$\mathrm{BASCH}$, Sophie (1991). Le Voyage imaginaire : les écrivains français en Grèce au XXe siècle. Athènes : Hatier ; BASCH, Sophie (1995). Le Mirage grec : la Grèce moderne devant l'opinion française depuis la création de l'École d'Athènes jusqu'à la guerre civile grecque, 1846-1946. Paris : Hatier. BOROWSKA, Małgorzata (2007). Filhellenizm w Polsce: rekonesans. Warszawa: Wydawnictwa Uniwersytetu Warszawskiego.

BOROWSKA, Małgorzata, KALINOWSKA, Maria, RÓżYCKA-TOMASZUK, Katarzyna (2012). Filhellenizm w Polsce: wybrane tematy. Warszawa : Wydawnictwa Uniwersytetu Warszawskiego. 
BRUNEAU, Michel (2001). « Peuples-monde de la longue durée : Grecs, Indiens, Chinois », L'Espace géographique, tome $30, \mathrm{n}^{\circ} 3,193-212$.

GAUTIER, Brigitte (2018). La poésie contre le chaos : une biographie de Zbigniew Herbert. Lausanne, Paris : Les Editions Noir sur Blanc.

GÉLY, Véronique (2009). «Les Anciens et nous : la littérature contemporaine et la matière antique ", Bulletin de l'Association Guillaume Budé 1, n $2: 19-40$. https://doi.org/10.3406/bude. 2009.2338 [consulté le 05/06/2020]

GÉLY, Véronique (2012). «Partages de l'Antiquité : un paradigme pour le comparatisme », Revue de littérature comparée 344, $\mathrm{n}^{\circ} 4: 387-395$. https://doi.org/10.3917/rlc.344.0387. [consulté le 05/06/2020]

HERBERT, Zbigniew (2000). Labirynt nad morzem. Warszawa : Fundacja Zeszytów Literackich ; HERBERT, Zbigniew, GAUTIER Brigitte (trad.) (2015). Le labyrinthe au bord de la mer. Paris : Le Bruit du temps.

JOCKEY, Philippe (2015). Le Mythe de la Grèce blanche : histoire d'un rêve occidental. Paris : Belin.

KUNDERA, Milan (1983). « Un Occident kidnappé : ou la tragédie de l'Europe centrale ». Le Débat 27, n 5 : 3. https://doi.org/10.3917/deba.027.0003 [consulté le 20/05/2020]

KUNDERA, Milan (2006). Le rideau : essai en sept parties. Paris : Gallimard.

LACARRIÈRE, Jacques (1984). L'Été grec : une Grèce quotidienne de 4000 ans. Nouv. éd. Revue, Corrigée et Augmentée d'une postface et de Débats critiques. Paris : Plon.

MASŁOWSKI, Michel, FRANCFORT, Didier, GRADVOHL, Paul (2011). Culture et identité en Europe centrale : canons littéraires et visions de l'histoire. Paris : Institut d'études slaves.

MONLUÇON, Anne-Marie, FIALKIEWICZ-SAIGNES, Anna (2016). Les Voyages en Europe des écrivains polonais (XIX ${ }^{e}-\mathrm{XXI}^{e}$ siècles). Grenoble : Université Grenoble Alpes.

SEFERIS, Georges, KOHLER, Denis (trad.) (1987). Essais : hellénisme et création. Paris: Mercure de France.

TZIOVAS, Dimitris (2014). Re-Imagining The Past, Antiquity and Modern Greek Culture. Oxford : Oxford University Press.

WESTPHAL, Bertrand (2007). La Géocritique : réel, fiction, espace. Paris : Les Éditions de Minuit.

YOURCENAR, Marguerite (1989). En pèlerin et en étranger : essais. Paris : Gallimard.

\section{RÉSUMÉS}

Longtemps resté imaginaire, le voyage en Grèce de Jacques Lacarrière et Zbigniew Herbert se concrétise en 1947 et 1964, dans un contexte historique qui rend la découverte de la "patrie imaginaire » incertaine et impérieuse à la fois. Guidés par le désir de confronter l'imaginaire au réel par le voyage, les deux auteurs se montrent soucieux de coucher sur le papier cette expérience tour à tour déroutante ou enthousiasmante. S'il prend parfois la forme d'un pèlerinage sur des lieux longtemps rêvés, étudiés et souvent idéalisés, le voyage réel est aussi l'occasion d'une redécouverte de la Grèce. Loin de l'imaginaire livresque, loin des représentations occidentales centrées sur l'Antiquité, la Grèce se révèle aux deux écrivains dans son histoire trimillénaire. Mais les voyages de Lacarrière et Herbert reflètent aussi, en retour, l'histoire européenne d'alors - entre guerre froide et remise en cause de la suprématie occidentale. 
Jacques Lacarrière's and Zbigniew Herbert's trips to Greece, which remained imaginary for a long time, take place in 1947 and 1964, in a historical context that makes the discovery of the "imaginary homeland" both uncertain and compelling. Guided by the desire to confront the imaginary with reality through travel, the two authors are also careful to give an account of their experience, which can be disconcerting or exciting. If it sometimes takes the form of a pilgrimage to places dreamed of, studied and often idealized, the real journey is also an opportunity to rediscover Greece. Far from the idealized representations usually centred around antiquity in Western culture, Greece reveals itself to the two writers in its three-thousand-year-old history. But Lacarrière and Herbert's travels also reflect the European history of the time - between the Cold War and the questioning of Western supremacy through decolonisation.

INDEX

Mots-clés : Grèce, récit de voyage, imaginaires, Lacarrière (Jacques), Herbert (Zbigniew)

Keywords : Greece, travel narrative, imaginaries, Lacarrière (Jacques), Herbert (Zbigniew)

\section{AUTEUR}

\section{MADELEINE SÉGUIER}

Sorbonne Université

madeleineseguier[at]live.fr 